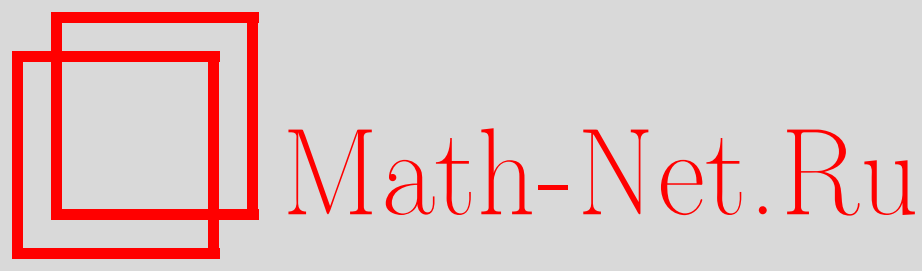

Н. А. Тюрин, Нестандартные лагранжевы торы и псевдоторические структуры, ТМФ, 2012, том 171, номер 2, 321-325

DOI: https://doi.org/10.4213/tmf6911

Использование Общероссийского математического портала Math-Net.Ru подразумевает, что вы прочитали и согласны с пользовательским соглашением http://www . mathnet.ru/rus/agreement

Параметры загрузки:

IP : 3.85 .5 .30

26 апреля 2023 г., 14:58:39

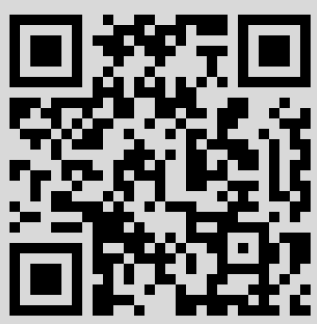


Том 171, № 2

май, 2012

(C) 2012 г. $\quad$ Н. А. Тюрин*†ђ

\title{
НЕСТАНДАРТНЫЕ ЛАГРАНЖКЕВ ТОРЫ И ПСЕВДОТОРИЧЕСКИЕ СТРУКТУРЫ
}

\begin{abstract}
Показано, что экзотические лагранжевы торы, построенные Чекановым и Шленком, могут быть получены на большом классе торических многообразий. Для этого их оригинальная конструкция переводится на язык псевдоторических структур. В качестве примера построены экзотические лагранжевы торы на поверхности дель Пеццо степени 6.
\end{abstract}

Ключевые слова: лагранжевы торы, экзотические торы, торы Чеканова, псевдоторическая структура.

\section{1. ВВЕДЕНИЕ}

Классификация лагранжевых торов (или, более общо, всевозможных лагранжевых подмногообразий) в симплектических многообразиях с точностью до симплектомофризма или гамильтоновой изотопии является важной математической задачей, решение которой требуется для многих подходов, принятых в математической физике. В настоящей работе мы исследуем скрученные торы [1], доставляющие примеры экзотических монотонных торов. Цепочка такого рода примеров начинается с тора $\Theta \in \mathbb{R}^{4}$, построенного в работах [2] и [3] в явном и неявном видах; тем самым была опровергнута гипотеза единственности класса монотонного тора в $\mathbb{R}^{4}$. С другой стороны, в работах [4], [5] была введена новая структура, обобщающая понятие торической структуры (такое обобщение естественно называть псевдоторической структурой), а также предложены примеры торических и неторических симплектических многообразий, обладающих такой структурой.

Настоящая работа представляет собой развернутый вариант краткой заметки [6], дополненный явными примерами нестандартных лагранжевых торов в торических поверхностях дель Пеццо. Мы показываем, что скрученный тор $\Theta^{k} \subset \mathbb{R}^{2 k+2}$, определенный в работе [1], может быть построен в терминах псевдоторической структуры. Вследствие того что всякая торическая поверхность дель Пеццо обладает псевдоторической структурой, оказывается возможным построить нестандартные торы в этих поверхностях.

*Объединенный институт ядерных исследований, Дубна, Московская обл., Россия. E-mail: ntyurin@theor.jinr.ru

${ }^{\dagger}$ Государственный университет - Высшая школа экономики, Москва, Россия

${ }^{\ddagger}$ Московский государственный университет путей сообщения (МИИТ), Москва, Россия 


\section{2. ПСЕВДОТОРИЧЕСКАЯ СТРУКТУРА НА $\mathbb{C}^{K+1}$ И СКРУЧЕННЫЙ ТОР $\Theta^{K}$}

Рассмотрим пространство $\mathbb{R}^{2 k+2}$ со стандартными комплексной и симплектической структурами и обозначим снаряженное пространство как $\mathbb{C}^{k+1}$. Выберем некоторую систему комплексных координат $\left(z_{1}, \ldots, z_{k+1}\right)$. Тогда можно определить отображение $\psi: \mathbb{C}^{k+1} \rightarrow \mathbb{C}$, задаваемое формулой

$$
\left(z_{1}, \ldots, z_{k+1}\right) \mapsto a=z_{1} \cdot \ldots \cdot z_{k+1} \in \mathbb{C} .
$$

Обозначим область значений этого отображения при фиксированной координате $a$ через $\mathbb{C}_{a}$. Слои отображения $\psi$ - гиперповерхности в $\mathbb{C}^{k+1}$, задаваемые уравнением $z_{1} \cdot \ldots \cdot z_{k+1}=a$. Эти поверхности являются гладкими за исключением случая $a=0$, а прообраз последнего состоит из $k+1$ базисных гиперповерхностей.

Стандартное торическое действие $T^{k+1}$ на $\mathbb{C}^{k+1}$ редуцируется до подтора $T_{0}^{k}$, действие которого сохраняет слои $\psi$. Соответствующее гамильтоново действие может быть описано в явном виде следующим образом. Возьмем набор самосопряженных операторов $A_{1}, \ldots, A_{k}$, задаваемых в нашей системе координат диагональными матрицами: $A_{i}=\operatorname{diag}\left(\lambda_{1}, \ldots, \lambda_{k+1}\right)$, где $\lambda_{i}=1, \lambda_{i+1}=-1$ и $\lambda_{j}=0$ для прочих $\lambda_{j}$. Рассмотрим функции $F_{i}=\left\langle A_{i} \psi, \psi\right\rangle_{h}$, известные в квантовой механике как средние значения. Тогда нетрудно видеть, что $\left\{F_{1}, \ldots, F_{k}\right\}$ - набор коммутирующих функций и каждая функция $F_{i}$ своим гамильтоновым действием сохраняет каждый слой $\psi$. Структура такого вида называется псевдоторической структурой (детальное определение см. в статье [5]).

Каждый гладкий слой $N_{a}=\psi^{-1}(a)$, снабженный набором функций $\left\{F_{1}^{a}, \ldots, F_{k}^{a}\right\}$, где $F_{i}^{a}=\left.F_{i}\right|_{N_{a}}$, есть вполне интегрируемая система [7]. Тогда выбор некритических значений $c_{1}, \ldots, c_{k}$ для функций $F_{1}^{a}, \ldots, F_{k}^{a}$ на гладком слое $N_{a}$ (т. е. $-1<c_{i}<1$ ) определяет совместное множество уровня

$$
S_{\left(c_{1}, \ldots, c_{k}\right)}^{a}=\left\{F_{i}^{a}=c_{i}\right\} \subset N_{a},
$$

которое является гладким лагранжевым тором для любого $a \neq 0$.

ЗАмечание 1 . Так как $F_{1}, \ldots, F_{k}$ - глобальные функции на $\mathbb{C}^{k+1}$, мы можем фиксировать значения $c_{1}, \ldots, c_{k}$ одновременно для всех слоев $N_{a}$ и всех интегралов $F_{1}^{a}, \ldots, F_{k}^{a}$. С другой стороны, некритические значения для $F_{i}$ и $F_{i}^{a}$ при $a \neq 0$ разные: для последних $c_{i}$ могут равняться нулю. Это отличие отражает тот факт, что критические точки функций $F_{1}, \ldots, F_{k}$ с нулевыми критическими значениями лежат в особом слое $N_{0}$.

ПреДЛОЖЕНИЕ 1. Въбор $c_{1}, \ldots, c_{k},-1<c_{i}<1$, в качестве значений для функицй $F_{1}^{a}, \ldots, F_{k}^{a}$ и гладкой петли $\gamma \subset \mathbb{C}_{a}$, не проходящей через ноль, порождает лагранжев тор в $\mathbb{C}^{k+1}$.

ДокАзАТЕЛЬство. Для всех $a \in \gamma \subset \mathbb{C}_{a}$ рассмотрим соответствующие торы $S_{\left(c_{1}, \ldots, c_{k}\right)}^{a}$ в слоях $\psi$ и объединим их:

$$
S_{\gamma,\left(c_{1}, \ldots, c_{k}\right)}=\bigcup_{a \in \gamma} S_{\left(c_{1}, \ldots, c_{n}\right)}^{a} ;
$$

очевидно, это даст гладкий тор $S_{\gamma,\left(c_{1}, \ldots, c_{n}\right)} \in \mathbb{C}^{k+1}$. 
Покажем, что этот тор лагранжев. Касательное расслоение $T S_{\gamma,\left(c_{1}, \ldots, c_{2}\right)}$ порождено $k+1$ не обращающимися в ноль векторными полями: гамильтоновыми векторными полями $X_{F_{i}}$ функций $F_{1}, \ldots, F_{k}$ и еще одним дополнительным векторным полем $\nabla_{\psi} \rho_{\gamma}$, являющимся результатом поднятия посредством симплектической связности $\nabla_{\psi}$ (напомним, что слои $\psi$ симплектические) не обращающегося в ноль касательного векторного поля $\rho_{\gamma}$ петли $\gamma$. Так как векторное поле $\nabla_{\psi} \rho_{\gamma}$ симплектически ортогонально слоям $\psi$, касательное пространство $T_{p} S_{\gamma,\left(c_{1}, \ldots, c_{n}\right)}$ лагранжево в каждой точке $p$.

Следуя Ору [8], мы различаем два случая:

1) стандартный тип - петля $\gamma$ не стягиваема в $\mathbb{C}_{a}^{*}=\mathbb{C}_{a}-\{0\}$;

2) тип Чеканова - петля $\gamma$ стягиваема в $\mathbb{C}_{a}^{*}$.

Такое различие основано на следующем наблюдении. Возьмем $c_{1}=\cdots=c_{k}=0$. Пусть $\gamma \in \mathbb{C}_{a}$ определена условием $\gamma_{0}=e^{i \phi}$. Тогда несложно понять, что $S_{\gamma_{0},(0, \ldots, 0)}-$ стандартный тор в $\mathbb{C}^{k+1}$. C другой стороны, параметризующее слои пространство $\mathbb{C}_{a}$ накрывает диагональ $\Delta \subset \mathbb{C}^{k+1}$, задаваемую условием $z_{1}=\cdots=z_{k+1}$, так что $z^{n+1}: \Delta \rightarrow \mathbb{C}_{a}$. Таким образом, пространство петель в $\mathbb{C}_{a}$ имеет компоненту, состоящую из петель следующего вида: возьмем сектор

$$
\mathcal{S}(k)=\left\{r e^{i \phi} \mid 0<\phi<\frac{2 \pi}{k+1}\right\} \in \Delta
$$

и рассмотрим такие петли в $\mathbb{C}_{a}$, которые получаются из петель, лежащих в секторе $\mathcal{S}(k)$, взятием $(n+1)$-й степени (ср. с работой [1]). Зафиксируем гладкую петлю $\gamma \subset \Delta$, лежащую в $\mathcal{S}(k) \cap D^{2}(k+1+\epsilon)$, как и в конструкции из работы [1]. Для каждой точки кривой $\gamma$ возьмем $(k+1)$-ю степень соответствующего комплексного числа и получим гладкую петлю $\gamma_{1} \in \mathbb{C}_{a}$. Тогда имеем

ПрЕДЛОЖЕНИЕ 2. В условиях предложения 1 возвмем $c_{1}=\cdots=c_{k}=0$ u nетлю $\gamma_{1}$, описанную выше. Тогда соответствующий лагранжсев тор $S_{\gamma_{1},(0, \ldots, 0)}$ совпадает с тором $\Theta^{k}$ из работы [1].

ДокАЗАТЕЛЬСтво получается прямым вычислением.

\section{3. ОБОБЩЕНИЯ}

В работе [1] конструкция экзотического тора $\Theta^{k}$ переносится на случай многообразий $\mathbb{C P}^{k}$ и $S^{2} \times \cdots \times S^{2}$. Оказывается, что и в этих случаях можно воспользоваться языком псевдоторических структур. Случай проективного пространства был подробно разобран в статье [5]; для прямого произведения $X=S_{1}^{2} \times \cdots \times S_{k}^{2}$ это может быть сделано следующим образом. Зафиксируем однородные координаты $\left[x_{j}: y_{j}\right]$ на $j$-м прямом слагаемом $S_{j}^{2}=\mathbb{C P}_{j}^{1}$ и рассмотрим отображение

$$
\psi: X-B \rightarrow S_{w}^{2}=\mathbb{C P}_{w}^{1},
$$

задаваемое соотношениями $w_{0}=x_{1} \cdot \ldots \cdot x_{k}, w_{1}=y_{1} \cdot \ldots \cdot y_{k}$. Здесь $B-$ базисное множество пучка

$$
\left(\mathbb{C P}_{w}^{1}\right)^{\vee}=\left\{\alpha x_{1} \cdot \ldots \cdot x_{k}+\beta y_{1} \cdot \ldots \cdot y_{k}=0, \quad \alpha, \beta \in \mathbb{C}\right\}
$$

на $X$. Тогда компактифицированный слой $D_{p}=\overline{\psi^{-1}(p)}$ является гладким дивизором в $X$, за исключением случаев, когда $p$ имеет координаты [0:1] или [1:0]. Этот 
слой является торическим многообразием с одним и тем же топологическим типом, кодируемым выпуклым многогранником $P$. Открытая часть $D_{p}-B$ расслоена на гладкие торы, и выбор внутренней точки в $P$ задает лагранжев тор в каждом слое $D_{p}$. Тогда для любой гладкой петли $\gamma \subset \mathbb{C P}_{w}^{1}$, не проходящей через северный и южный полюсы многобразия $\mathbb{C P}_{w}^{1}$, получаем гладкий лагранжев тор в $X$. Снова имеются два случая: петля $\gamma$ либо не стягиваема в многообразие $\mathbb{C P}_{w}^{1}$ с выколотыми полюсами (стандартный тип), либо стягиваема (тип Чеканова). Нетрудно видеть, что эти случаи в точности соответствуют примеру из работы [1].

Кроме того, более сложные скрученные торы, обозначаемые в работе [1] через $\Theta_{l}^{k}$, могут быть построены в рамках теории псевдоторических структур. Дело в том, что слои $\phi$ в наших построениях снова являются торическими многообразиями, и в них можно рассматривать не стандартные торы $S_{\left(c_{1}, \ldots, c_{k}\right)}$, а экзотические. Тогда мы получим абсолютно такую же древесную иерархию, как в работе [1]. Однако в свете возможности построения псевдоторической структуры на произвольном торическом многообразии (набросок доказательства этого факта см. в статье [5], полную версию мы планируем привести в готовящейся публикации) лагранжевы торы типа $\Theta_{l}^{k}$ существуют в произвольных торических многообразиях, и древесная иерархия имеется также в этом общем случае. При этом открытым остается вопрос о различении этих торов в задаче классификации лагранжевых торов с точностью до гамильтоновой изотопии.

В конце нашего рассмотрения приведем пример псевдоторической структуры и нестандартных лагражевых торов на торической поверхности дель Пеццо. В работе [1] поставлен вопрос о нестандартных торах в $\mathbb{C P}_{3}^{2}-$ проективной плоскости с тремя раздутыми точками. Именно этот случай мы и рассмотрим.

ПримеР 1. Проективная плоскость $\mathbb{C P}_{3}^{2}$ с тремя раздутыми точками моделируется как полное пересечение в $\mathbb{C P}_{x}^{2} \times \mathbb{C P}_{y}^{2}$, задаваемое уравнениями

$$
U=\left\{x_{0} y_{0}=x_{1} y_{1}=x_{2} y_{2}\right\}
$$

при этом каноническая проекция "сдутия" $\pi: \mathbb{C P}_{3}^{2} \rightarrow \mathbb{C P}^{2}$ получается просто путем проецирования на любой из прямых сомножителей.

Рассмотрим отображение $\psi: \mathbb{C P}_{x}^{2} \times \mathbb{C P}_{y}^{2}-B \rightarrow \mathbb{C P}_{w}^{1}$, задаваемое соотношениями $w_{0}=x_{0} x_{1} y_{2}^{2}, w_{1}=x_{2}^{2} y_{0} y_{1}$. Ограничим $\psi$ на $U \subset \mathbb{C P}_{x}^{2} \times \mathbb{C P}_{y}^{2}$, исключая базисное множество $B_{U}=B \cap U$, состоящее из шести прямых вида $x_{i}=x_{j}=y_{k}=0$ или $x_{i}=y_{j}=y_{k}=0$, где $i, j, k$ - произвольный набор неодинаковых чисел из множества $\{0,1,2\}$. Обозначим такое ограничение как $\psi_{U}: U-B_{U} \rightarrow \mathbb{C P}_{w}^{1}$. Нетрудно видеть, что компактифицированные слои отображения $\psi_{U}$ являются дивизорами на $U$ вида $B_{U} \cup \Sigma_{p}$, где $p \in \mathbb{C P}_{w}^{1}$. В самом деле, каждый компактифицированный слой есть пересечение $U$ и соответствующего элемента пучка

$$
\left\{-w_{1}(p) x_{0} x_{1} y_{2}^{2}+w_{0}(p) x_{2}^{2} y_{0} y_{1}=0\right\}
$$

Очевидно, что пересечение всегда содержит множество $B_{U}$, однако для каждой точки $p$ с ненулевыми координатами $\left[w_{0}(p): w_{1}(p)\right]$ имеется еще одна компонента $\Sigma_{p}$, соответствующая квадрике

$$
Q_{x}=\left\{\sqrt{-w_{1}(p)} x_{0} x_{1}+\sqrt{w_{0}(p)} x_{2}^{2}=0\right\} \subset \mathbb{C P}_{x}^{2} .
$$


Найдем гладкую функцию $f$ на $U$, которая сохраняет слои отображения $\psi_{U}$. Искать будем, как всегда, в виде

$$
f=f\left(\lambda_{i} ; \mu_{j}\right)=\frac{\sum \lambda_{i}\left|x_{i}\right|^{2}}{\sum\left|x_{i}\right|^{2}}+\frac{\sum \mu_{i}\left|y_{i}\right|^{2}}{\sum\left|y_{i}\right|^{2}} .
$$

Условие сохранения множества $U$ при инфинитезимальном действии гамильтонова поля такой функции дает

$$
\lambda_{0}+\mu_{0}=\lambda_{1}+\mu_{1}=\lambda_{2}+\mu_{2},
$$

и без ограничения общности мы можем положить $\lambda_{i}=-\mu_{i}$. Отсюда можно получить две коммутирующие алгебраически независимые функции на $U$, например функции $f_{1}=\left.f(1,-1,0 ;-1,1,0)\right|_{U}$ и $f_{2}=\left.f(0,1,-1 ; 0,-1,1)\right|_{U}$, которые вместе дадут отображения действия $F_{\text {act }}: U \rightarrow P$ в выпуклый шестиугольник $P \subset \mathbb{R}^{2}$. Но нам нужно из этих двух функций оставить только одну, которая сохраняет слои отображения $\psi_{U}$. Такая функция должна удовлетворять условиям

$$
\lambda_{0}+\lambda_{1}+2 \mu_{2}=2 \lambda_{2}+\mu_{0}+\mu_{1}=0,
$$

отсюда $2 \lambda_{2}=\lambda_{1}+\lambda_{2}$. Этому условию удовлетворяет только функция $f_{1}$, она и будет функцией $f$, сохраняющей слои отображения $\psi_{U}$. Заметим, что $f$ имеет на множестве $U$ только невырожденные критические точки, расположенные в вершинах базисного шестиугольника $B_{U}$; критические значения $f$ равны $2,1,1,-1,-1,-2$. Отсюда следует, что компонента $\Sigma_{p}$ гладкая вне $B_{U}$ за исключением северного и южного полюсов многообразия $\mathbb{C P}_{w}^{1}$.

Подводя итог, получаем псевдоторическую структуру $\left(f=f_{1}, \psi_{U}\right)$ на торической поверхности дель Пеццо $U=\mathbb{C P}_{3}^{2}$. И снова, выбирая петлю $\gamma \subset \mathbb{C P}_{w}^{1}$, не проходящую через северный и южный полюсы, и значение функции $f \neq \pm 2$, мы получаем лагранжев тор, тип которого определяется тем, стягиваема или нет петля $\gamma$ в многообразие $\mathbb{C P}_{w}^{1}$ с выколотыми полюсами.

Благодарности. Работа выполнена при поддержке Лаборатории алгебраической геометрии Государственного университета - Высшая школа экономики, поддержана грантом Правительства РФ (договор № 11.G34.31.0023), а также РФФИ (гранты № 10-01-93113, 11-01-00980_а).

\section{Список литературы}

[1] Yu. Chekanov, F. Schlenk, Electron. Res. Announc. Math. Sci., 17 (2010), 104-121.

[2] Yu. V. Chekanov, Math. Z., 223:4 (1996), 547-559.

[3] Ya. Eliashberg, L. Polterovich, "The problem of Lagrangian knots in four-manifolds", Geometric Topology: 1993 Georgia International Topology Conference (University of Georgia, Athens, GA, August 2-13, 1993), AMS-IP Studies in Advanced Mathematics Series, ed. W. H. Kazez, AMS, Cambridge, MA, 1993, 323-327.

[4] С. А. Белев, Н. А. Тюрин, Матем. заметки, 87:1 (2010), 48-59.

[5] Н. А. Тюрин, ТМФ, 162:3 (2010), 307-333.

[6] Н. А. Тюрин, УМН, 66:1(397) (2011), 185-186.

[7] Н. Н. Нехорошев, Функи. анализ и его прил., 28:2 (1994), 67-69.

[8] D. Auroux, J. Gökova Geom. Topol. GGT, 1 (2007), 51-91. 